Voix et Images

voixetimages

\title{
La fonction sociale de la poésie
}

Jacques Pelletier

Volume 19, numéro 1 (55), automne 1993

Lionel Groulx écrivain

URI : https://id.erudit.org/iderudit/201078ar

DOI : https://doi.org/10.7202/201078ar

Aller au sommaire du numéro

\section{Éditeur(s)}

Université du Québec à Montréal

\section{ISSN}

0318-9201 (imprimé)

1705-933X (numérique)

Découvrir la revue

\section{Citer cet article}

Pelletier, J. (1993). La fonction sociale de la poésie. Voix et Images, 19(1),

188-197. https://doi.org/10.7202/201078ar d'utilisation que vous pouvez consulter en ligne.

https://apropos.erudit.org/fr/usagers/politique-dutilisation/ 


\section{La fonction sociale de la poésie}

Jacques Pelletier, Université du Québec à Montréal

Deux ouvrages ${ }^{1}$, parus récemment, soulèvent avec vigueur et rigueur la question fort controversée du statut et de la fonction sociale du discours poétique. Langage spécialisé, travail d'exploration et de recherche à partir des mots communs de la tribu détournés de leur usage courant, destiné généralement à un public restreint et privilégié de "connaisseurs", le texte poétique apparaît affecté le plus souvent d'une aura singulière, d'un coefficient d'ineffabilité qui lui permettrait d'échapper en quelque sorte aux déterminations sociales qui régissent la langue et par suite à l'analyse tant de sa genèse concrète, matérielle, que de son usage, son rôle dans le monde réel des hommes et de l'histoire.

Chacun à sa manière, à partir de points de vue différents, les ouvrages de Pierre Popovic et de François Dumont interrogent ce lieu commun, fondement du curieux univers de croyance dans lequel baigne la poésie et qui en fait un lieu cantonné dans des frontières étanches, un champ clos réservé à quelques bappy few touchés par la grâce et préservé des soucis du commun des mortels. 
C'est à partir de la théorie du discours social, mise au point par Marc Angenot, que Pierre Popovic attaque son objet constitué par trois recueils particulièrement révélateurs, véritables emblèmes marquant autant de bornes dans l'évolution du discours poétique québécois, dans son passage de la tradition à la modernité: Étal mixte de Claude Gauvreau, Deux Sangs de Gaston Miron et Le Tombeau des rois d'Anne Hébert, publiés en 1953. Écrits et édités durant la *grande noirceur * duplessiste, ces recueils seraient traversés et imprégnés dans leur substance même par la conjoncture politique et culturelle de l'époque et de manière plus directe, plus immédiate, par le discours social du temps, dominé par la pensée de Lionel Groulx et de ses disciples. C'est l'hypothèse centrale de l'ouvrage.

Il s'agit donc pour Popovic de lire et d'expliquer ces textes à la lumière de ce qu'il appelle les principales "formations discursives" (revues, mouvements sociaux et culturels) de la période qui trouvent elles-mêmes leur signification dans leur rapport au "groulxisme". L'analyse sociocritique est de la sorte couplée, intégrée à la théorie du discours social dans une démarche méthodologique qui comprend deux étapes, deux moments, sinon deux objets: la reconstitution du discours hégémonique de la période considérée, suivie de l'analyse critique des recueils retenus.

Le "groulxisme" est considéré par Popovic comme l'horizon idéologique le plus englobant du discours social de la période qui va de 1937 à 1955. La doctrine du chanoine lui semble la synthèse la plus achevée de la pensée traditionnelle dominante au Canada français, reprenant en termes plus contemporains l'essentiel du discours messianique mis au point par les forces clérico-nationalistes dans la deuxième moitié du siècle précédent: conserver l'héritage (français et catholique) de la collectivité et assumer sa vocation spirituelle en Amérique du Nord, sa tâche principale et son destin, toujours à accomplir.

Le duplessisme, pour sa part, apparaît comme un prolongement naturel, sur le plan politique, du groulxisme. Il garde l'essentiel de l'armature idéologique de la doctrine, en fait un usage abondant et répétitif dans les discours publics qui servent de rituels liturgiques à ses pratiques et à ses politiques qui, elles, sont toutefois souvent beaucoup plus "modernes" que les palabres qui les accompagnent et les encadrent. Cette interprétation du duplessisme comme phénomène essentiellement progressiste sur le plan économique, sinon social et culturel, due aux travaux récents d'historiens et de sociologues (Boismenu, Bourque et Duchastel, entre autres) est reprise par Popovic qui 
fait du "cheuf " une sorte de moderne malgré lui. Interprétation originale bien sûr, à contre-courant de l'historiographie traditionnelle qui en faisait un chef de clan réactionnaire et despotique, mais qui peut laisser sceptique surtout lorsqu'on songe par exemple à l'état de la culture sous le règne du vieux célibataire hargneux de Trois-Rivières.

Quoi qu'il en soit, c'est par rapport au groulxisme et à son culte de ce que l'auteur appelle le aroman historique épique - nœud de la doctrine - et au duplessisme, son incarnation contemporaine, que les forces nouvelles, surgissant dans la conjoncture sociale et culturelle des années 1940, auront à se définir. La Relève, fondée en 1934, en pleine crise économique, reprend par exemple la vision du monde chrétienne, idéaliste - du chanoine, mais s'en démarque sur le plan politique - elle se méfie du nationalisme - et culturel - elle se réclame des "modernes". Cité libre, créée en 1949, par des journalistes, des universitaires, des syndicalistes, rompt pour sa part avec la vision du monde globalisante et unitaire du groulxisme et adopte une perspective fonctionnaliste, privilégiant l'analyse empirique, sectorielle, de la réalité contemporaine. Il en va de même des universitaires des facultés des sciences sociales qui étudient le Québec moderne sans recourir au filtre de l'idéologie nationaliste dominante et qui se réclament plutôt des avancées de la réflexion américaine dans ce secteur. La même observation vaut pour des disciplines plus spéculatives comme la philosophie ou la psychologie qui, tout en demeurant à l'intérieur de la perspective chrétienne sur le monde, tentent d'intégrer les apports nouveaux de l'existentialisme et du freudisme notamment.

Ces courants modernes s'affirment tous, cependant, à partir du discours social hégémonique de la période et le reprennent, d'une manière ou d'une autre, même lorsqu'ils semblent s'en démarquer de manière très ostensible: l'ancien couve sous le nouveau, le nourrit, et d'une certaine façon le détermine. Ainsi, pour Popovic, le discours automatiste, insurrectionnel, révolutionnaire de Refus global participe, par exemple, "de la même hégémonie discursive" que le groulxisme, dont il reprendrait le "modèle historico-épique" (p. 2) et il véhiculerait finalement pour l'essentiel la même vision du monde. Ce serait aussi le cas, mais à un moindre degré, pour La Nouvelle Relève et Cité libre qui, dans cette optique, seraient cependant plus « modernes " que Refus global, ce qui, à première vue, semble pour le moins étonnant et prêter à discussion. J'y reviendrai plus loin.

Cette reconstruction historique effectuée, l'auteur se livre à l'analyse des recueils retenus à la lumière de l'horizon discursif ainsi dégagé. Les textes sont donc placés en situation, dans le cadre plus 
général de l'ensemble des discours qui circulent à l'époque, qu'ils reprennent et infléchissent.

Étal mixte fait l'objet d'un travail particulièrement soigné. Popovic consacre en effet au texte de Gauvreau son analyse la plus approfondie, la plus développée, la plus stimulante aussi. Il met en lumière, de manière convaincante, l'intrication dans le recueil de l'ancien présence du religieux, de la liturgie et de ses rites - et du nouveau le marché, la place publique, la bruyante ville moderne -, l'intégration remarquable, la fusion dans une même coulée textuelle de tout l'ensemble des sociolectes qui expriment aussi bien la réalité montréalaise du temps - français normatif du collège classique, langage populaire de la rue - que l'état du discours poétique contemporain que Gauvreau utilise en tentant de le "révolutionner " par la pratique de l'xexploréen*. Cela donne une assez extraordinaire incarnation du fameux plurilinguisme décrit par Bakhtine, si bien qu'Étal mixte " paraît avoir été écrit sous l'égide ${ }^{2}$ " du théoricien russe!

Au terme d'analyses remarquables qu'il serait trop long de rappeler ici, Popovic conclut que la poésie de Gauvreau, en 1951, au moment d'Étal mixte, "oscille entre le pluriloque et le soliloque, entre le carnavalesque et la seule intronisation d'un roi déchu, entre une position libérale et un putsch terroriste, entre l'ouverture à l'autre et la fermeture sur soi $^{3}{ }^{n}$. Oscillation qui sera d'ailleurs constante par la suite, tout au long de l'expérience et de l'œuvre du poète.

L'analyse des recueils de Miron et d'Hébert est plus "courte ", tant sur le plan qualitatif que quantitatif. Après l'extraordinaire et entrầnante démonstration opérée sur le texte de Gauvreau, Popovic semble tout à coup manquer de souffle. Il évoque de manière beaucoup plus cursive, en effet, Deux Sangs de Miron qui, à l'époque, est animateur d'un groupe de folklore et qui vient tout juste d'arriver en ville, à Montréal. Ses textes sont empreints à la fois de la tradition à travers leur nostalgie de l'univers rassurant et homogène de la campagne et du moderne, en ce qu'ils expriment, sur le mode du malaise, de l'inconfort, la réalité nouvelle et bougeante de la ville. Miron, soutient Popovic, sait toutefois que la solution avancée par le groulxisme est anachronique, qu'elle ne saurait donc être une réponse crédible aux problèmes du présent et il la cherche alors plutôt du côté de l'utopisme communautaire: créer des réseaux d'amitié, d'entraide, de partage à travers notamment les rencontres que favorise la poésie lui paraît une solution souhaitable.

Le Tombeau des rois est également étudié à la lumière de la situation de l'auteure dans la configuration idéologique de l'époque. Anne 
Hébert apparaît dans le sillage de Cité libre, participant de son discours réformiste et néo-libéral: à sa manière toute personnelle, par l'importance accordée à la vie intime, personnelle, à une thématique amoureuse à connotation nettement érotique bien que voilée, elle met en cause la société traditionnelle et notamment le discours masculin fortement chauvin qui caractérise la période. Popovic va jusqu'à conclure que "Ille Tombeau des rois valorise donc un esprit d'entreprise qui s'arc-boute sur une assomption de la subjectivité et sur l'émergence d'un nouveau sujet communautaire. Le néo-libéralisme du début des années 1950 , précise-t-il, ne fait pas autre chose ${ }^{4}$. Si l'on veut... mais on ne peut s'empêcher de penser que l'extrapolation, ici, est sans doute excessive. Cela dit, malgré l'intérêt réel et la consistance de l'analyse, l'étude du recueil d'Hébert, comme celle de Deux Sangs, n'a pas la profondeur ni l'acuité de celle effectuée sur Gauvreau qui paraît davantage portée par la passion, et parfois, par une volonté polémique, la perspective y étant nettement plus critique qu'à l'endroit d'Anne Hébert et de Gaston Miron.

En somme, on ne saurait trop attirer l'attention sur cet ambitieux ouvrage, et ce pour plusieurs raisons. Il est l'un des premiers à tenter concrètement une articulation entre la théorie toute récente du discours social et une sociocritique, déjà pas très vieille, qui s'était essentiellement cantonnée jusque-là à l'analyse du discours romanesque. Il accorde ensuite l'intérêt qu'elle mérite à une période trop peu étudiée de l'histoire culturelle et littéraire du Québec, et plus encore dans le sous-champ de la poésie que dans le reste. Il met cette production en relation avec le discours social hégémonique de la période et les contre-discours ou les discours parallèles qui surgissent alors, menaçant de l'intérieur l'hégémonie discursive. Sur ce plan, son travail de reconstitution historique est considérable et très bien informé, y compris lorsque ses interprétations prêtent à discussion. Enfin, cette démonstration est opérée à partir de l'analyse minutieuse, détaillée, approfondie de trois textes-charnières particulièrement représentatifs de *l'esprit du temps *.

Cela étant, il reste que l'ouvrage soulève des questions et appelle parfois des réserves. On peut se demander par exemple si la théorie du discours social est bien, comme postulé ici, la voie royale de la sociocritique contemporaine, ce qui est loin d'être évident. Je ne suis pas du tout convaincu pour ma part qu'il y ait de tels liens naturels et quasi obligés entre ces deux disciplines qui relèvent de préoccupations fort différentes, d'ordre historique pour la théorie du discours social, d'ordre d'abord littéraire pour la sociocritique. 
On peut interroger également la fonction explicative du discours social par rapport aux textes étudiés: en rend-il compte vraiment, y compris sur le plan de leurs caractéristiques formelles, proprement esthétiques? Popovic affirme que oui, bien sûr, mais sa démonstration est-elle toujours convaincante? Le rapport établi entre Hébert et le néo-libéralisme évoqué plus haut, et ce n'est qu'un exemple, n'entraîne pas forcément l'adhésion (ça sent un peu le "forcing "!) quoiqu'on pense par ailleurs de la qualité des propositions concernant tant l'analyse des textes que le discours social: ce qui pose problème, c'est leur mise en rapport, la vertu explicative qu'il faut lui accorder.

On peut, sur un autre plan, ne pas toujours partager les jugements de l'auteur, notamment sur Gauvreau et Refus global. Popovic, assez curieusement, est plutôt * dur " pour les automatistes qui apparaissent dans ses analyses plus près du groulxisme - dont ils reprendraient le romanesque historico-épique - que La Nouvelle Relève et Cité libre. Ce qui l'amène à proposer une typologie étrange, et pour le moins discutable, où l'on retrouve du côté de la Tradition le groulxisme et à sa manière Refus global, du côté du Moderne, La Nouvelle Relève et Cité libre et quelque part à mi-chemin entre ces deux pôles: le duplessisme! Disons que cette répartition, ce classement peut laisser à tout lé moins songeur sinon carrément sceptique.

Enfin, le statut même du groulxisme comme horizon idéologique en quelque sorte indépassable pourrait aussi être discuté. Sur cette base, Popovic se trouve à mettre l'accent davantage sur ce qui continue que sur ce qui bouge durant cette période, sur la tradition plutôt que sur l'innovation et la rupture (qui ne saurait être qu'une fausse rupture, attestant tout simplement de la profonde emprise du vieux et de l'ancien, d'un déjà-là insurmontable).

Ces questions que l'ouvrage soulève, ces discussions qu'il appelle, ce sont autant de marques de qualité qui signalent le caractère stimulant d'une interprétation qui mérite d'être débattue, le débat permettant aussi d'avancer dans la compréhension d'une période et des biens symboliques qu'elle produit de quelque manière, y compris lorsqu'il s'agit des productions poétiques les plus achevées et les plus hautes: elles n'échappent pas non plus au poids de l'Histoire qui les fait et qu'elles font dans une incessante dialectique. 
-La littérature a-t-elle une fonction ${ }^{5}$ ?, se demande très explicitement François Dumont dans l'ouverture de son ouvrage consacré aux Usages de la poésie. C'est là, selon lui, la grande question qui préoccupe les poètes québécois durant l'après-guerre et qui ne cessera de refaire régulièrement surface jusqu'au début de la décennie 1970. Son propos est d'examiner comment les poètes dans leurs écrits sur la littérature, l'art, la culture, ont formulé cette question et quelles réponses ils lui ont apportée.

Le corpus examiné est donc constitué pour l'essentiel de textes de réflexion, à dimension parfois manifestaire, et non de poèmes. Ce qui est au centre des analyses, c'est donc le discours et non les pratiques des poètes convoqués, étant implicitement entendu qu'il peut y avoir entre les uns et les autres des oscillations, des écarts plus ou moins importants que l'étude, toutefois, ne prend pas en compte.

Dumont retient la période 1945-1970 comme cadre de sa recherche. Il découpe à l'intérieur de celle-ci trois sous-périodes: la première (1945-1952) recouvre des phénomènes aussi divers que La Nouvelle Relève, Cité libre et Refus global; la deuxième (1953-1963) est marquée essentiellement par l'apparition et l'établissement de l'Hexagone; la troisième (1963-1970) est dominée par Parti pris et la problématique de l'engagement. Il s'agit de montrer comment, à l'intérieur de chacun de ces "moments *, les poètes, et les groupes plus ou moins informels auquels ils appartiennent, conçoivent la poésie. L'approche est donc résolument historique et, il faut le dire, assez classique, ce qui n'est pas pour moi un "défaut", bien au contraire.

Dumont fait bien voir que dans l'immédiate après-guerre la poésie est d'abord conçue comme expérience spirituelle. Pour les animateurs de La Nouvelle Relève, elle est en quelque sorte un révélateur de l'âme et l'expression la plus haute d'une culture dont les fondements sont divins. La question de la fonction de l'art n'est donc pas abordée à partir de la problématique de l'engagement, comme le fait Sartre, par exemple, à l'époque, mais sur un plan plus général, détaché des contingences immédiates: la littérature fait partie de l'expérience humaine, elle témoigne de profondes aspirations à la vérité et à la grandeur, mais elle échappe par cela même aux déterminations des conjonctures culturelles et politiques qui forment la trame du court terme historique. Refus global qui, pour Dumont, est proche de La Nouvelle Relève en ce qu'il partage le même topos de l'unité, se singularise toutefois par le questionnement explicite qu'il formule à son endroit: fondé sur l'imaginaire, le désir, le rêve, l'absolu, l'art appelle une révolution globale tant des individus que de la culture; en cela il n'est 
pas conçu comme une pratique spécifique, autonome, à l'écart du monde mais bien comme l'expression même de la vie. Sa perspective, sur ce plan, le rapproche davantage de la problématique des avantgardes que de celle des "modernes", qu'il fera sienne plus tard, après la perte des illusions sur le pouvoir transformateur de l'art.

Avec l'Hexagone, s'ouvre une nouvelle période dominée par la question des rapports entre poésie et projet national (qui n'a pas encore, bien sûr, sa forme indépendantiste): en quoi, comment la poésie est-elle partie prenante de l'aventure collective? C'est à cette interrogation que tentent de répondre, en ordre disposé, les poètes du temps. Et si la problématique est commune, les réponses, elles, sont parfois fort différentes. Gaston Miron est partagé entre une conception de la poésie comme témoignage, lieu d'un engagement et la tentation du silence devant son impuissance à exercer une action réelle sur l'histoire. Michel van Schendel est hostile à son engagement explicite, tout en considérant qu'elle est fondamentalement liée au procès historique. Brault et Fernand Ouellette se méfient de tout ce qui pourrait ressembler à un embrigadement. Il reste que chez tous, fait remarquer fort pertinemment Dumont, la question des rapports poésie/société (et projet national) est posée dans des termes dynamiques, relevant de ce qu'il appelle un topos dialectique.

Parti pris, au début des années soixante, reprend ce topos et le radicalise. Revue à vocation d'abord politique et sociale, mais fondée par des hommes de culture et de littérature, elle ne peut pas ignorer cette question du statut et du rôle de l'art en société. Contrairement à ce qu'on serait porté à penser, elle n'a toutefois pas de réponse toute faite et bien arrêtée dans ce débat. Paul Chamberland, par exemple, hésite, oscille, partagé entre une position prônant l'engagement immédiat et une autre le retrait, croyant par moments à l'efficacité sociale, politique, de la poésie et se montrant à d'autres moments sceptique, incertain de ses pouvoirs réels. Cette dernière position pourra même prendre la forme du retrait, du silence (chez un Hubert Aquin et un Gaston Miron à certaines périodes). À l'inverse, l'autre position trouvera à s'exprimer dans des textes aussi engagés que L'afficheur burle, préfiguration de la poésie militante des années soixante-dix.

Dumont rappelle avec finesse la richesse des discussions et des débats qui eurent alors lieu à la revue et dans ses environs, alimentés par des pensées aussi riches et diverses que l'existentialisme, le marxisme et l'anticolonialisme en pleine effervescence (théorique et pratique). Son tableau, tout en nuances, s'oppose à l'image stéréotypée, unifiée et réductrice, que l'on présente parfois des positions 
artistiques et littéraires de Parti pris, complétant heureusement, dans le cas de la poésie, le portrait plus global proposé par Robert Major dans son ouvrage de synthèse consacré à ce mouvement ${ }^{6}$.

Au tournant des années soixante-dix, la problématique de Parti pris éclate suite à la disparition de la revue en 1968. De nouveaux discours apparaissent, allant du refus radical de toute fonction sociale à la poésie, chez les formalistes de La Barre du jour, par exemple, à son intégration la plus complète, à sa fusion dans l'expérience la plus quotidienne, la plus immédiate chez les adeptes de la contre-culture, dont Chamberland devient un héraut, ou encore à son embrigadement total au service du politique, comme ce sera le cas un temps chez Charron. S'ouvre ainsi une nouvelle période que Dumont, à la suite de bien d'autres, qualifie de postmoderne et dans laquelle la question du rôle social de l'art et de la poésie est débattue désormais dans la perspective d'une " fin de l'histoire".

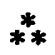

L'ouvrage de Dumont, comme celui de Popovic, projettent une image éclairante sur une période cruciale dans l'évolution de la poésie contemporaine au Québec. À partir de préoccupations et de méthodes différentes (théorie du discours social et sociocritique pour l'un, histoire pour l'autre), ils posent tous les deux, à l'occasion d'un cas concret, l'importante question de la place, du statut et de la fonction de l'art en société.

C'est là une question pas nouvelle, bien sûr, dans le champ littéraire, mais rarement posée dans le domaine spécifique de la poésie. C'est l'intérêt théorique de leurs ouvrages qui, en outre, s'offrent comme des analyses de textes particulièrement réussies (dans le cas de Popovic) et des monographies accomplies (sur le discours social des années quarante, chez Popovic, sur le discours des poètes chez Dumont). Avec leurs contributions, la sociocritique et la sociologie de la littérature, accusées à juste titre de trop souvent ignorer la poésie, accomplissent un pas en avant. Ce n'est pas un mince mérite.

1. Pierre Popovic, La Contradiction du poème. Poésie et discours social au Québec de 1948 à 1953, Candiac, Les Éditions Balzac, coll. «L'Univers des discours?, 1992, 460 p.; François Dumont, Visages de la poésie. Le discours des poètes québécois sur la fonction de la poésie, Québec, Les Presses de l'Université Laval, coll. *Vie des lettres québécoises :, 1993, 250 p. 
2. Pierre Popovic, op. cit., p. 175.

3. Ibid., p. 300 .

4. Ibid., p. 389.

5. François Dumont, op. cit., p. 1.

6. Robert Major, Parti pris: idéologies et littérature, Montréal, Hurtubise HMH, 1979. 